\title{
Afasia e Alteração de Comportamento: Desafio Diagnóstico
}

Aphasia and Behavior Disorders: Clinical Challenge

Sandra Afonso ${ }^{1,2}$, Mariana Nogueira1 ${ }^{1}$, José Carlos Ferreira ${ }^{1,2}$, Ana Serrão Neto ${ }^{1}$

Autor Correspondente:

Sandra Afonso [sandra.afonso@jmellosaude.pt]

R. Mário Botas, 1998-018 Lisboa, Portugal

\section{RESUMO}

INTRODUÇÃO: A síndrome de Landau-Kleffner é caracterizada por uma afasia adquirida, associada a atividade paroxística contínua no sono, documentada no eletroencefalograma. Descreve-se este caso com o objetivo de alertar para a importância da suspeição clínica deste diagnóstico.

CASO CLÍNICO: Criança do sexo feminino, com desenvolvimento psicomotor prévio adequado, inicia aos 5 anos quadro de alteração do comportamento, acompanhada de regressão da linguagem, compreensiva e expressiva. Após suspeição clínica, o diagnóstico de síndrome de Landau-Kleffner foi confirmado pelo eletroencefalograma, com atividade paroxística centro-temporal bilateral de predomínio esquerdo e padrão de ponta-onda contínua durante o sono. Iniciou terapêutica farmacológica com corticoide, antiepilético e terapia da fala. Verificou-se melhoria do comportamento e linguagem, com normalização do eletroencefalograma.

DISCUSSÃO: A síndrome de Landau-Kleffner pode apresentar variantes no tipo de manifestações clínicas iniciais, podendo mimetizar algumas doenças do foro psiquiátrico. A precocidade no diagnóstico e intervenção tem implicações no prognóstico a longo termo.

PALAVRAS-CHAVE: Afasia; Criança; Eletroencefalografia; Síndrome Landau-Kleffner; Transtornos do Desenvolvimento da Linguagem

1. Centro da Criança e do Adolescente, Hospital CUF Descobertas, Lisboa, Portugal.

2. Unidade de Neurodesenvolvimento, Centro da Criança e do Adolescente, Hospital CUF Descobertas, Lisboa, Portugal.

Recebido: 03/04/2018 - Aceite: 06/06/2018 


\section{ABSTRACT}

INTRODUCTION: The Landau-Kleffner syndrome is characterized by acquired aphasia with paroxistic activity with sleep pattern, in the electroencephalogram. The aim of this case report is to show the importance of a strong clinical suspect to achieve this diagnosis.

CASE REPORT: A girl, with previous normal development, at 5-years-old underwent a behavioral disorder and language regression, comprehensive and expressive. The diagnosis of Landau-Kleffner syndrome was confirmed by electroencephalogram, with bilateral centro-temporal paroxistic activity, with potentiation of spikes during the sleep. She started pharmacological treatment, with antiepileptic and corticosteroids, and speech therapy. The patient showed an improvement of behavior and language, with a normal electroencephalogram.

DISCUSSION: The Landau-Kleffner syndrome may have different initial presentations that may lead to misdiagnosis and confusion with psychiatric disorders. Early diagnosis and intervention have implications for long-term prognosis.

KEYWORDS: Aphasia; Child; Electroencephalography; Landau-Kleffner Syndrome; Language Development Disorders

\section{INTRODUÇÃO}

A síndrome de Landau-Kleffner (SLK), ou afasia epilética adquirida, é uma síndrome neurológica rara, de etiologia desconhecida, embora fatores genéticos possam estar envolvidos, nomeadamente uma mutuação no gene GRIN2A. ${ }^{1}$ Caracteriza-se por regressão da linguagem numa criança previamente saudável, associada ao aparecimento de atividade paroxística contínua no sono no eletroencefalograma (EEG). ${ }^{2-5}$

O quadro inicia-se, mais comummente, entre 3 e 8 anos,

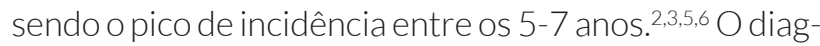
nóstico é baseado nas alterações clínicas e eletroencefalográficas. A afasia está sempre presente e pode desenvolver-se em semanas ou meses e, muitas vezes, é a primeira manifestação clínica. 2,3,6 Os testes neuropsicológicos revelam uma perturbação da descodificação fonológica como défice primário, a que se associa, em consequência, a afasia adquirida de expressão. ${ }^{3,5}$ Pode apresentar uma evolução flutuante, variar com períodos de remissão parcial espontânea e agravamento. Pode ainda associar-se a alterações do comportamento e sintomas psiquiátricos, incluindo irritabilidade, agressividade, défice de atenção e sintomas sugestivos de perturbação do espectro do autismo. ${ }^{3}$ Os episódios epiléticos manifestam-se, na maioria dos casos, como crises epiléticas focais, seguindo-se em frequência crises tónico-clónicas generalizadas e de ausência.3,4 Corresponde a cerca de 0,2\% de epilepsias da infância. ${ }^{4}$

O EEG mostra a presença de atividade paroxística temporal ou parieto-occipital bilateral, com padrão ponta-onda potenciada pelo sono, que deverá estar presente em mais de $85 \%$ do traçado de sono não REM. ${ }^{2,7}$

O diagnóstico diferencial pode ser muito extenso, e inclui défice auditivo, alterações específicas da linguagem, perturbações do espectro do autismo, encefalopatias epileptiformes e doenças psiquiátricas como perturbação desintegrativa da infância.

O tratamento farmacológico inclui corticoterapia e antiepiléticos, como valproato de sódio e diazepam. ${ }^{8} \mathrm{O}$ tratamento cirúrgico não é consensual, uma vez que apenas permite controlo das crises epiléticas quando refratárias, e não a recuperação dos restantes sintomas, que na maioria são bem controlados com tratamento ou outras intervenções como terapia da fala, psicologia ou tratamento psiquiátrico.

De acordo com alguns relatos das últimas duas décadas, os sintomas de apresentação inicial parecem variar da descrição original desta patologia, em 1957 por Landau e Kleffner, o que dificulta a correta identificação e determinação da real prevalência.3,6 Um estudo epidemiológico no Japão, mostrou uma incidência de SLK de 1/100 0000 em crianças dos 5 aos 14 anos de idade, sendo a prevalência nas crianças dos 5 aos 19 anos sob terapêutica médica de 1/300 000 a 1/410 000.8,9 A descrição deste caso pretende mostrar as principais características desta síndrome, cujo diagnóstico é determinado pela suspeita clínica, que sendo precoce, tem importantes implicações no prognóstico.

\section{CASO CLÍNICO}

Apresenta-se uma criança do sexo feminino, raça caucasiana, atualmente com 7 anos de idade. Na linha materna a referir antecedentes familiares de epilepsia, perturbação do desenvolvimento da linguagem e perturbação de aprendizagem específica.

A gravidez foi vigiada, sem complicações. Nasceu às 38 semanas, de cesariana, índice de Apgar de 9 ao primeiro minuto e 10 ao quinto minuto. $\bigcirc$ rastreio de doenças 
metabólicas foi normal. Período perinatal decorreu sem intercorrências. $\bigcirc$ crescimento estaturoponderal foi adequado e adquiriu os principais marcos do desenvolvimento psicomotor na idade esperada: sentou-se sem apoio aos 7 meses, gatinhou aos 9 meses, teve marcha independente aos 12 meses, disse as primeiras palavras com significado antes dos 12 meses, fez frases aos 2 anos, adquiriu o controlo dos esfíncteres anal e vesical no segundo ano de vida. Ingressou no infantário aos 6 meses, não tendo sido relatadas dificuldades na adaptação.

Aos 5 anos de idade iniciou um quadro de alteração progressiva do comportamento, com dois meses de evolução, caracterizada por agitação psicomotora, birras, episódios de fúria com atitudes de auto, e mais tarde, também hetero-agressão, conduzindo a isolamento social. Acompanhou-se de perturbação do sono, insónia terminal, assim como de regressão da linguagem, com dificuldade em responder ao nome e a perguntas simples. Era referida como "parecendo que não ouvia" (sic). Foram negados episódios sugestivos de crises epiléticas, história de traumatismo craniano ou de infeção recente. Foi observada na unidade de neurodesenvolvimento, objetivando-se agitação psicomotora, que dificultava a exploração funcional. Estabelecia pouca interação com o adulto, chamando a atenção através do toque, alternando entre o alheamento e uma atitude de desafio, não cumprindo ordens e atirando objetos para chão quando contrariada. Ao nível da linguagem, apresentava redução da expressão verbal espontânea, com perturbação na articulação verbal, vocabulário pobre e respostas descontextualizadas.

A avaliação formal e completa do desenvolvimento psicomotor não foi possível por incapacidade de colaboração, mas revelou afasia de expressão e compreensão. Salientava-se a incapacidade na descodificação da mensagem verbal, tal como em nomear e identificar objetos, reconhecendo-os pela função. O raciocínio lógico parecia preservado. $\bigcirc$ restante exame neurológico era normal.

Perante um quadro clínico progressivo de afasia e alteração do comportamento, colocou-se a hipótese diagnóstica de síndrome de Landau-Kleffner. Para confirmar, realizou EEG que mostrou atividade paroxística centro-temporal bilateral (Fig.s 1 e 2) e um padrão de ponta-onda contínua do sono (Fig. 3). A ressonância magnética cranioencefálica não mostrou alterações estruturais. O rastreio auditivo foi normal, excluindo défice auditivo.

Iniciou terapêutica com valproato de sódio (15 mg/kg/ dia), prednisolona (1 mg/kg/dia) e melatonina (1 mg/ dia). A ocorrência de duas convulsões tónico-clónicas generalizadas obrigou ao aumento da dose do valproato

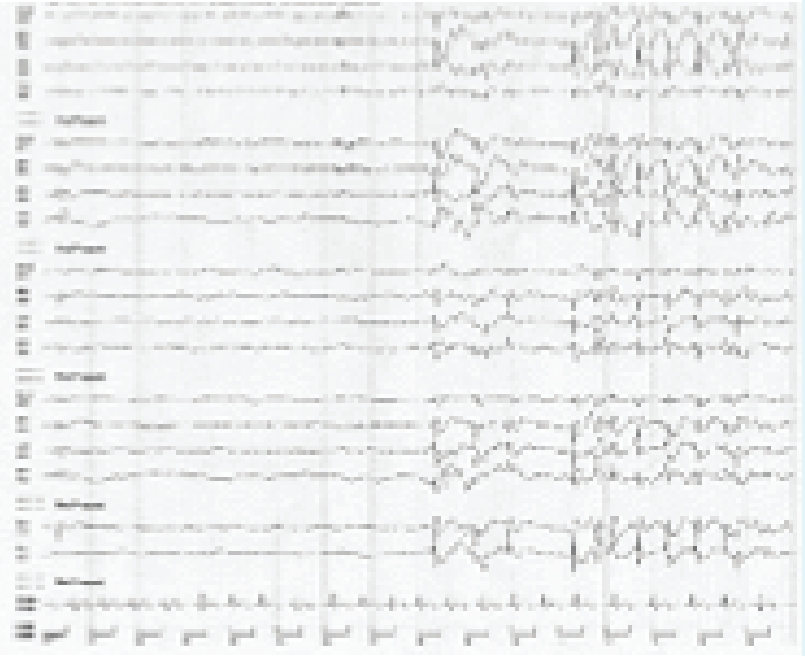

FIGURA 1. Eletroencefalograma com atividade paroxística centro-temporal bilateral (vigília).

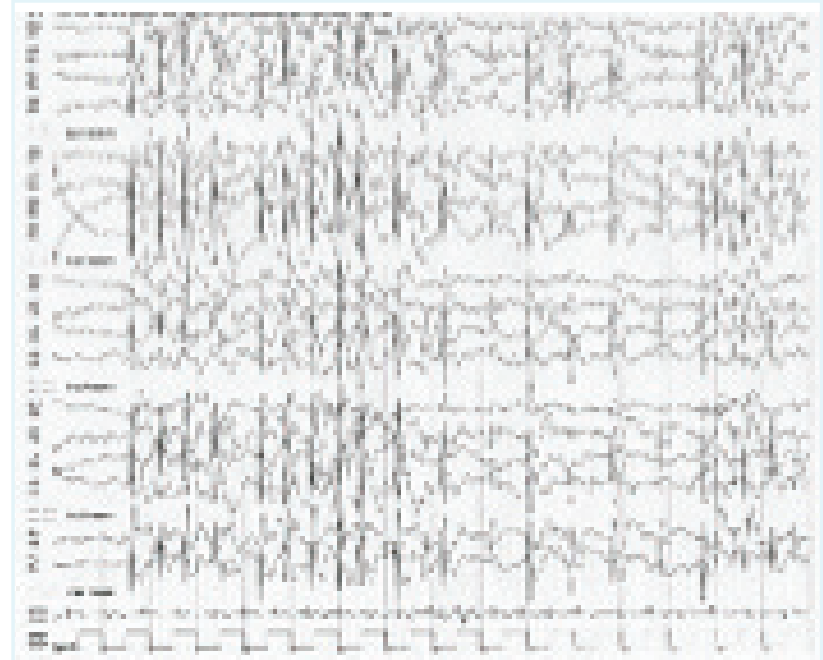

FIGURA 2. Eletroencefalograma com atividade paroxística centro-temporal bilateral (vigília)

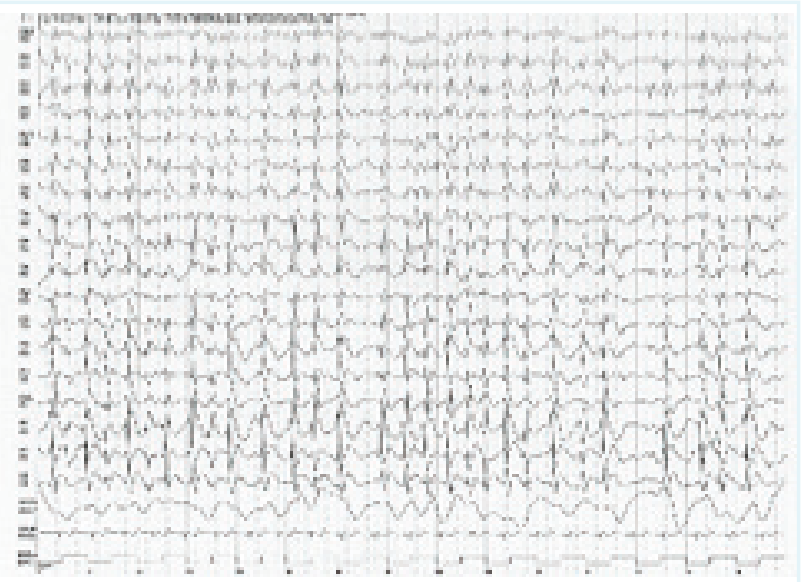

FIGURA 3. Eletroencefalograma com padrão de ponta-onda contínua do sono.

para 20 mg/kg/dia, sem crises posteriores e desaparecimento de atividade paroxística no EEG. Aos 6 meses de corticoterapia houve recuperação súbita da linguagem verbal, pelo que diminuiu progressivamente a dose até suspender, tendo completado um ano. 
Ingressou numa escola mista, de crianças deficientes auditivas, com recurso a linguagem gestual, assegurando um percurso escolar adequado, se a evolução clínica fosse desfavorável. Por queixas de marcada desatenção e agitação, perturbando o comportamento e desempenho na sala de aula, foi associado o metilfenidato $(1 \mathrm{mg} / \mathrm{kg} /$ dia), favorecendo a aprendizagem e reintegração escolar. Em termos de intervenção começou Terapia da Fala, que ainda mantém com uma frequência semanal.

Aos dois anos de evolução da doença, verifica-se uma melhoria do quadro comportamental, ao nível da irrequietude e sociabilização. Na linguagem expressiva apresentou dificuldades na articulação verbal, que está a recuperar, formando atualmente frases de modo espontâneo e sem agramatismos. Tem-se registado uma melhoria a nível lexical, com conversação e relato de vivências mais pormenorizado. São ainda evidentes limitações na compreensão da linguagem oral, nomeadamente nos segundos sentidos das palavras, como ironia, absurdos ou situações de humor. Iniciou o primeiro ano de escolaridade com 7 anos de idade, que decorre sem aparentes dificuldades de aprendizagem.

\section{DISCUSSÃO}

Este caso descreve um exemplo de SLK numa criança de 5 anos, do sexo feminino, que se apresentou inicialmente com sintomas, predominantemente, comportamentais, nomeadamente agressividade e isolamento social, mimetizando uma perturbação psiquiátrica.

A SLK é uma forma de afasia adquirida em crianças com adequado desenvolvimento prévio da linguagem. Dentro das síndromes do espectro epilepsia-afasia, é uma patologia rara e é duas vezes mais comum no sexo masculino. ${ }^{4} \mathrm{O}$ diagnóstico da SLK pode ser, de facto, um desafio clínico, principalmente nos casos que não se associam a crises epiléticas. Quando estas são a primeira manifestação, ajudam a estabelecer o diagnóstico, mas até cerca de 1/3 dos pacientes pode mesmo não desenvolver epilepsia. ${ }^{4,10}$ Além disso, tal como no caso descrito, as alterações do comportamento podem constituir o primeiro sintoma. Estas podem incluir sintomas de agressividade, alterações do sono, apatia e regressão global social, podendo alternar entre períodos de extrema apatia e outros de comportamento hiperativo/agressivo. Estas alterações podem levar a um atraso ou erro de diagnóstico. Nestes casos, a SLK deve ser diferenciada de doenças psiquiátricas, como transtorno bipolar. As alterações específicas da linguagem não incluem alterações no EEG e geralmente as crianças não apresentam desenvolvimento da linguagem inicial normal.
No que respeita ao quadro de perturbação do espectro do autismo (PEA), as principais diferenças residem na idade de regressão da linguagem, em média mais tardia na SLK, quando grande parte do vocabulário está já adquirido, na ausência de dificuldades na interação social ou de comportamentos restritivos. Embora possa existir isolamento social, este é consequência da incapacidade de comunicar. Neste caso, a afasia surgiu primariamente com um défice de compreensão a que se seguiu a perda quase global da linguagem expressiva, mantendo-se preservadas as habilidades não-verbais.

diagnóstico baseia-se na suspeição clínica, confirmada pelo padrão de ponta-onda contínua característico do EEG no sono. Os exames de neuroimagem, não mostram anomalias estruturais características, ${ }^{6,7}$ tal como neste caso. Com o recurso à medicina nuclear, nomeadamente com tomografia por emissão de positrões, podem ser encontradas alterações como hipometabolismo nas áreas temporais, compatível com a disfunção cerebral da área correspondente. ${ }^{6}$ Um estudo da análise do volume cerebral de quatro crianças com SLK, mostrou uma atrofia cortical focal bilateral nas áreas temporais superiores, desconhecendo-se se é a causa do SLK ou consequência da atividade epileptiforme refratária. ${ }^{5,7}$

Os corticoides em altas doses constituem a primeira linha de tratamento, verificando-se melhoria tanto clínica (linguagem e comportamento) como eletroencefalográfica. ${ }^{4}$ No que respeita às crises epiléticas, raramente são graves e respondem ao tratamento com antiepiléticos convencionais, em regra até à idade adulta. 5,10 Existe ainda uma abordagem cirúrgica, a secção subpial múltipla, cujos resultados são controversos. ${ }^{6,10}$

O prognóstico de crianças com SLK é variável. Em algumas pode persistir perturbação da linguagem de forma permanente. No entanto, é mais frequente alguma recuperação, especialmente com intervenção adequada. Quanto mais cedo surgem os sintomas causados pela interrupção da normal maturação dos lobos temporais num período crítico e precoce de formação e "pruning" de sinapses, mais grave será a evolução a longo termo. ${ }^{7}$ Assim, o prognóstico é melhor quando o início do quadro ocorre após os 6 anos e a intervenção com terapia da fala é iniciada precocemente. ${ }^{11}$ Se não existir remissão completa da afasia, a evolução pode ser para atraso moderado a grave da linguagem na vida adulta, podendo estar presente até cerca de metade dos casos. ${ }^{5}$

Em conclusão, pode ser difícil distinguir casos da SLK de outras situações que incluem regressão da linguagem e especialmente se associada a alterações do comportamento. No entanto, nos casos clássicos de SLK o dese- 
jo de comunicar está mantido e a criança é socialmente responsiva, como se verificou no caso descrito. ${ }^{10}$ Nesta fase do desenvolvimento, o reconhecimento precoce pode influenciar consideravelmente o prognóstico.

CONFLITOS DE INTERESSE: Os autores declaram não ter qualquer conflito de interesse na realização do presente trabalho.

FONTES DE FINANCIAMENTO: Não houve qualquer fonte de financiamento na realização do presente trabalho.

CONFIDENCIALIDADE DOS DADOS: Os autores declaram ter seguido os protocolos da sua instituição acerca da publicação dos dados de doentes.

PROTEÇÃO DE PESSOAS E ANIMAIS: Os autores declaram que os procedimentos seguidos na elaboração do presente trabalho estão em conformidade com as normas das comissões de investigação clínica e de ética, bem como da declaração de Helsínquia e da Associação Médica Mundial.

CONFLICTS OF INTEREST: The authors declare that they have no conflicts of interest.

FINANCIAL SUPPORT: This work has not received any contribution, grant or scholarship.

CONFIDENTIALITY OF DATA: The authors declare that they have followed the protocols of their work center on the publication of data from patients.

PROTECTION OF HUMAN AND ANIMAL SUBJECTS: The authors declare that the procedures followed were in accordance with the regulations of the relevant clinical research ethics committee and with those of the Code of Ethics of the World Medical Association (Declaration of Helsinki).

\section{REFERÊNCIAS}

1. Myers KA, Scheffer IE. GRIN2A-Related Speech Disorders and Epilepsy. In: Adam MP, Ardinger HH, Pagon RA, Wallace SE, Bean LJ, Stephens K, et al, editors. SourceGeneReviews ${ }^{\circledR}$. Seattle: University of Washington; 2018.

2. Landau-kleffner Syndrome [homepage na Internet]. International League Against Epilepsy. [consultado em maio de 2018]. Disponível em: https://www.epilepsydiagnosis.org.

3. Baumer FM, Cardon AL, Porter BE. Language dysfunction in pediatric epilepsy. J Pediatr. 2018; 194: 13-21. doi: 10.1016/j. jpeds.2017.10.031.

4. Campos JG, Guevara LG. Landau-Kleffner. J Pediatr Neurol. 2007;5:93-9.

5. Bourgeois BF, Landau WM. Landau-Kleffner syndrome and temporal cortical volume reduction. Neurology. 2004;63:1152-3

6. Alonso AJ. El síndrome de Landau-Kleffner. Presentación de dos casos. Rec Cubana Pediatr 2005;77:835-9.

7. Lee YJ, Hwang SK, Kwon S. The Clinical spectrum of benign epilepsy with centro-temporal spikes: a challenge in categorization and predictability. J Epilepsy Res. 2017; 7:1-6. doi: 10.14581/jer.17001.

8. Munckhof BV, Alderweireld C, Davelaar S, Teeseling VH, Nikolakopoulos S, Braun KPJ, et al. Treatment of electrical status epilepticus in sleep: Clinical and EEG characteristics and response to 147 treatments in 47 patients. Eur J Paediatr Neurol. 2018; 22:64-71. doi: 10.1016/j.ejpn.2017.08.006.

9. Kaga M, Inagaki M, Ohta R. Epidemiological study of Landau-Kleffner syndrome (LKS) in Japan. Brain Dev. 2014;36:284-6.

10. Ribeiro KMN, Assumpção FB, Valente KD. Síndrome de Landau-Kleffner e regressão autística. Arq Neuropsiquiatr. 2002;60:835-9.

11. Palaré MJ, Gomes AL. Síndrome de Landau-Kleffner e epilepsia de ausências- uma associação rara. Acta Pediatr Port. 2004:2:135-8. 